\title{
Causal reasoning over knowledge graphs leveraging drug-perturbed and disease-specific transcriptomic signatures for drug discovery
}

\author{
Daniel Domingo-Fernández ${ }^{1, *}$, Yojana Gadiya ${ }^{1}$, Abhishek Patel ${ }^{1}$, Sarah Mubeen ${ }^{2}$, Daniel Rivas-Barragan ${ }^{3}$, \\ Chris W. Diana ${ }^{1}$, Biswapriya B. Misra ${ }^{1}$, David Healey ${ }^{1}$, Joe Rokicki ${ }^{1}$, Viswa Colluru ${ }^{1, *}$
}

1. Enveda Biosciences, Boulder, CO, 80301, USA

2. Bonn-Aachen International Center for IT, Rheinische Friedrich-Wilhelms-Universität Bonn, Bonn 53115, Germany

3. Barcelona Supercomputing Center, Barcelona 08034, Spain

*Corresponding Authors: Viswa Colluru and Daniel Domingo-Fernández. Enveda Biosciences, Boulder, CO, 80301, USA. viswa.colluru@envedabio.com and daniel.domingo-fernandez@envedabio.com

Keywords: knowledge graphs; network medicine; network biology; causal reasoning; drug discovery

\begin{abstract}
Network-based approaches are becoming increasingly popular for drug discovery as they provide a systems-level overview of the mechanisms underlying disease pathophysiology. They have demonstrated significant early promise over other methods of biological data representation, such as in target discovery, side effect prediction and drug repurposing. In parallel, an explosion of -omics data for the deep characterization of biological systems routinely uncovers molecular signatures of disease for similar applications. Here, we present RPath, a novel algorithm that prioritizes drugs for a given disease by reasoning over causal paths in a knowledge graph (KG), guided by both drug-perturbed as well as disease-specific transcriptomic signatures. First, our approach identifies the causal paths that connect a drug to a particular disease. Next, it reasons over these paths to identify those that correlate with the transcriptional signatures observed in a drug-perturbation experiment, and anti-correlate to signatures observed in the disease of interest. The paths which match this signature profile are then proposed to represent the mechanism of action of the drug. We demonstrate how RPath consistently prioritizes clinically investigated drug-disease pairs on multiple datasets and KGs, achieving better performance over other similar methodologies. Furthermore, we present two applications showing how one can deconvolute the predictions made by RPath as well as predict novel targets. Finally, we have made the source code and data publicly available at https://github.com/enveda/RPath.
\end{abstract}




\section{Introduction}

The representation of biomolecular interactions occurring within cells is often intuitively organized in the form of biological networks. These networks can be used to inherently model biological processes through the use of nodes denoting biological entities and edges representing their relationships. While homogeneous networks, such as protein-protein interaction networks, can represent relationships between a single entity type, knowledge graphs (KGs) can incorporate a broad range of biological scales, from the genetic and molecular level (e.g., proteins, drugs, and biochemicals), to biological concepts (e.g., phenotypes and diseases). These KGs can then can be utilized for several applications in drug discovery, such as providing insights into molecular mechanisms and therapeutic targets (Fotis et al., 2018; Bharadhwaj et al., 2021), side effect prediction in the early stages of drug development (Zitnik et al., 2018), target prioritization (Sang et al., 2018), and drug repositioning (Nelson et al., 2019).

Given the flexibility of KGs, multiple heterogeneous relation types can be modeled to represent biological processes that are governed by interactions occurring between component entities (Bonner et al., 2021). Even though a variety of relation types (e.g., literature co-occurrence, associations, etc.) can be leveraged by network-topology algorithms for various applications, causal relations are particularly useful as they can be used to infer the effect of any given node on another by reasoning over the KG (MacLean, 2021). Nonetheless, not all interactions included in a given KG are necessarily biologically relevant as they may be context-specific, such as to a particular cell type, tissue or disease. Furthermore, as the complete human interactome remains unknown, KGs modeling PPIs are also incomplete and the interactions which are modeled tend to be biased towards well studied proteins and their relationships (Schaefer et al., 2015). One approach to address these challenges is to jointly leverage prior knowledge in KGs with data-driven -omics experiments (Liu et al., 2019; Belyaeva et al., 2021; Winkler et al., 2021).

Experimental datasets have been widely employed by recent drug repurposing approaches to identify drug candidates for a given disease using the anti-correlation in biological processes or pathways at the transcriptomic- or proteomic- level between drugs and diseases as a proxy (Iorio et al., 2010; Sirota et al., 2011; Peyvandipour et al., 2018; Emon et al., 2020) (see Samart et al., 2021 for a recent review). While these approaches use prior knowledge in the form of pathways (gene sets), this concept has yet to be applied on KGs for drug discovery. However, by mapping the signatures of an -omics experiment to a $\mathrm{KG}$, we can not only verify which causal interactions are observed within a specific context, but also prioritize and identify the mechanism of action of a drug for a given disease with high precision.

Currently, there exist numerous algorithms that leverage causal relations for the interpretation of -omics data. In general, these algorithms operate by assessing the concordance between transcriptomic or proteomic signatures and the predicted causal effects encoded in these relations (Hill et al., 2017; Babur et al., 2021). For instance, the Reverse Causal Reasoning (RCR) (Catlett et al., 2013) and Network Perturbation Amplitude (NPA) algorithms 
(Martin et al., 2012; Martin et al., 2014) assess and score this concordance employing causal graphs consisting of up-stream and down-stream proteins (nodes) representing regulations occurring in biological pathways. Subsequently, the scores obtained from these algorithms can be used for the interpretation of -omics data commonly derived from contrast experiments. Although the interpretations obtained from these algorithms may be relevant for several downstream applications, such as drug target prediction, disease characterization, and side effect prediction, the algorithms themselves cannot be directly used for these applications. Additionally, these algorithms have been specifically designed for bipartite graphs, thus, simplifying biological pathways to a single relation between two proteins.

While traditionally, these algorithms were applied on small causal networks, they have recently begun to be applied on large-scale KGs, given the increasing availability of causal information, including proteins, drugs and phenotypes. For instance, a recent algorithm we published, drug2ways, reasons over all paths between a drug and a disease in a KG to predict the effect of the drug as the cumulative effect of all directed interactions between these two nodes (Rivas-Barragan et al., 2020). Reasoning over all paths overcomes the limitation of earlier algorithms that exclusively account for shortest paths on protein-protein interaction networks, oversimplifying the effect exerted by one node on another, as all other paths between the two nodes are ignored (Chindelevitch et al., 2012; Krämer et al., 2014). Nonetheless, paths in large-scale KGs can grow exponentially, many of which may not be relevant in a true biological context. Thus, incorporating signatures from context-specific experimental datasets along with prior knowledge in a KG can enable us to reason over the entire network-structure and ensure only paths which can be observed in a biologically meaningful context are retained. In doing so, we can address several of the limitations of the above-mentioned methods for drug discovery.

Here, we present RPath, a novel algorithm that prioritizes drugs for a particular disease by reasoning over causal paths in a KG, guided by both drug-perturbed and disease-specific transcriptomic signatures. We demonstrate how RPath is able to recover a large proportion of clinically investigated drug-disease pairs on multiple transcriptomic datasets and KGs, performing better than other network-based methods. Furthermore, we show two additional applications where we illustrate how our approach can also assist in hypothesis generation and target prioritization.

\section{Methods}

\subsection{Theoretical background}

We denote a $\mathrm{KG}$ as a set of nodes and edges, where nodes correspond to three distinct biological entities (i.e., chemicals, proteins, and diseases) connected through causal relations, representing activatory or inhibitory effects. Causal relations within the KG connect drug-protein, protein-protein, and protein-disease nodes. A (directed) path in a $\mathrm{KG}$ is defined as a sequence of two or more biological entities connected through causal relations. Paths in the $\mathrm{KG}$ can be either cyclic or simple. A cyclic path refers to paths in which one or more nodes repeat, whereas a simple 
path corresponds to a path in which no nodes appear more than once. The length of a path is defined by the number of edges that connect the nodes within the path.

\subsection{RPath algorithm}

The algorithm used in our framework, RPath, reasons over the paths in a KG to identify all possible effects a given drug can have on a disease (Figure 1). Each of these paths can be divided into three main sequential parts that attempt to represent the mechanism of action of a drug: i) the drug activates/inhibits a protein target (drug-protein edge), ii) the protein target triggers a signaling cascade (a set of protein-protein edges), and iii) the signaling cascade reverts the disease condition (protein-disease edge). Furthermore, since every causal edge contains information on the effect each node exerts on another (i.e., activation or inhibition), we can infer the direction of regulation (i.e., up-/down-regulated) for each node at each step of a path (Chindelevitch et al., 2012; Krämer et al., 2014).

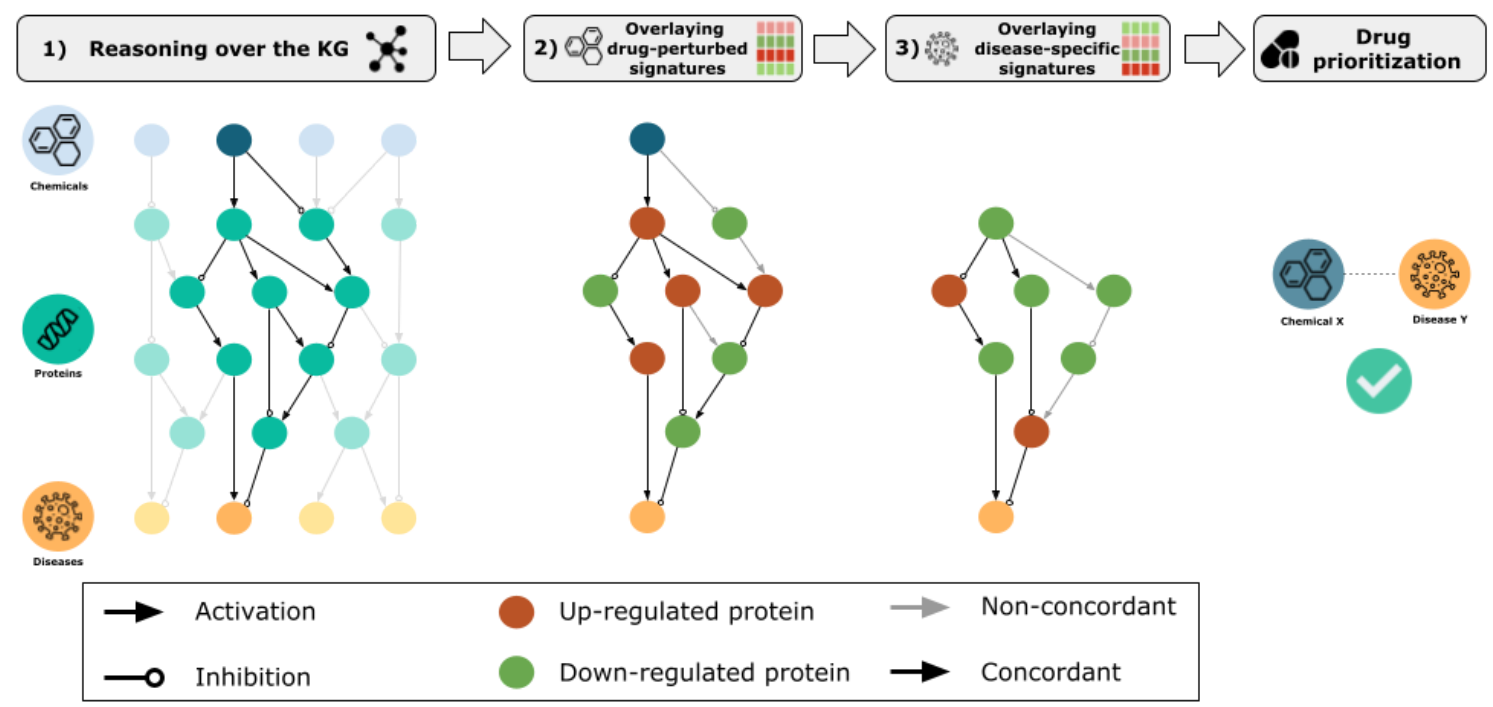

Figure 1. Schematic representation of the RPath algorithm. Step 1) All paths of a given length between a drug and a disease in the KG are calculated. If there exists a causal acyclic path connecting the drug and the disease, a subgraph involving all these paths is inferred. This subgraph represents the proposed mechanism of action by which the drug might be a therapeutic target of the given disease. Step 2) Transcriptomic signatures observed from a drug-perturbed experiment are overlaid onto each corresponding node present in these paths. Then, RPath traverses through each path and evaluates whether the inferred direction of regulation (i.e., activation or inhibition) at every step is concordant with the upand down- regulations (i.e., red and green nodes, respectively) observed in the transcriptomic signatures. Finally, non-concordant paths are removed. Step 3) In a similar manner, transcriptomic signatures observed within a specific disease context are overlaid onto each node in the concordant paths from the previous step (if any). Next, RPath evaluates whether the disease transcriptomic signatures contradict the paths that were concordant with the drug signatures. If this is the case, the specific drug-disease pair is prioritized.

Once the causal acyclic paths between a particular drug and disease in the KG have been calculated (Figure 1; step 1), the next step of RPath is to overlay transcriptomic signatures from a drug-perturbed experiment (Figure 1; step 2). We hypothesize that because a number of paths might represent the biologically relevant mechanism of action of this drug, the observed transcriptomic signatures for proteins in the KG should be concordant with the inferred up- or down-regulations at every step of the path. For example, if in a given path, a drug inhibits a protein 
target and that target activates a signaling cascade, we expect the inhibition of the protein target as well as the inhibition of the proteins downstream of the target. We would like to note that a gene is considered to be differentially expressed if its expression is significantly altered with respect to a reference sample (i.e., control). Keeping this in mind, a cut-off is applied to each measured gene in the experimental dataset based on the fold change; this measurement is used to define whether the gene is up-/down-regulated or unchanged.

Similarly, the final step of RPath involves overlaying disease-specific transcriptomic signatures to the nodes in the paths of the KG (Figure 1; step 3). We hypothesize that, in contrast to the overlaying of drug-perturbed signatures, transcriptomic signatures in a disease context should be anti-correlated to both the drug-perturbed signatures as well as the inferred up- or down-regulations for every node in the path. This final step is inspired by previous work that exploited the anti-correlation between drug and disease signatures at the pathway level for drug repurposing (Peyvandipour et al., 2018; Emon et al., 2020). In summary, RPath aims at prioritizing a specific drug for a given disease if i) there exist causal paths between the drug and disease in the $\mathrm{KG}$, ii) the causal effects on these paths are aligned with the transcriptomic changes observed in the drug-perturbed experiment, and iii) both the drug signatures and the paths are anti-correlated with the transcriptomic dysregulations observed in the disease. Figure 2 outlines the pseudocode of the described logic of the algorithm.

As an additional application, the algorithm can be modified following the same logic for target prioritization (see Supplementary Figure 1 for the pseudocode). This variant of the algorithm begins from a disease of interest and calculates all paths from the disease to all proteins for a given path length (e.g., a path length of 6). Next, it calculates the concordance between the paths for each potential protein target and the transcriptomic signatures of the given disease to assess whether there are proteins that could be key up-stream regulators of the observed phenotype. We would like to note that this application significantly increases the running time of the algorithm as it requires querying paths from a disease to several thousands of proteins in the $\mathrm{KG}$, as opposed to only a handful of chemicals. 


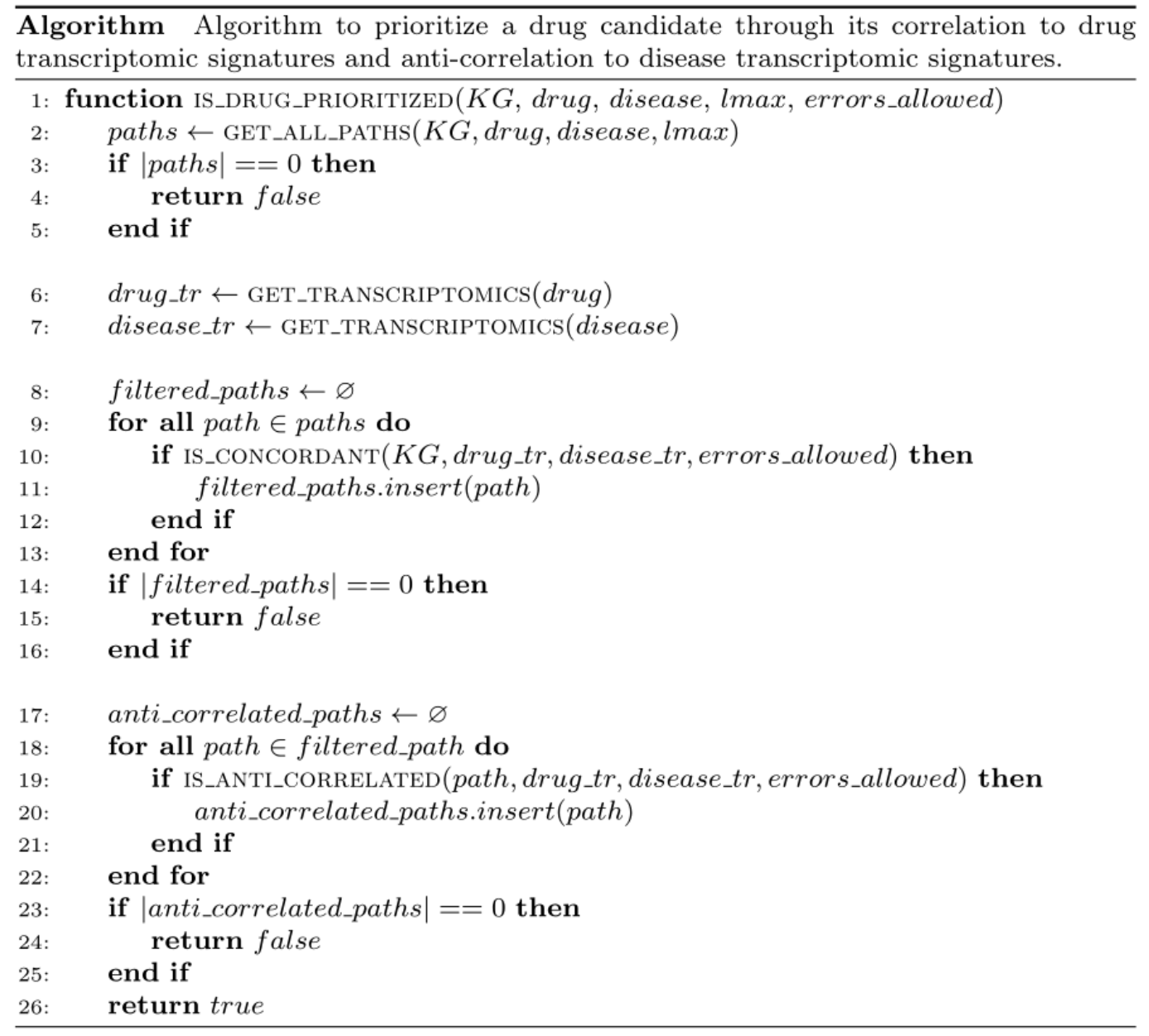

Function 1 Assess whether the path between a drug and a disease is concordant with the observed drug transcriptomic signatures

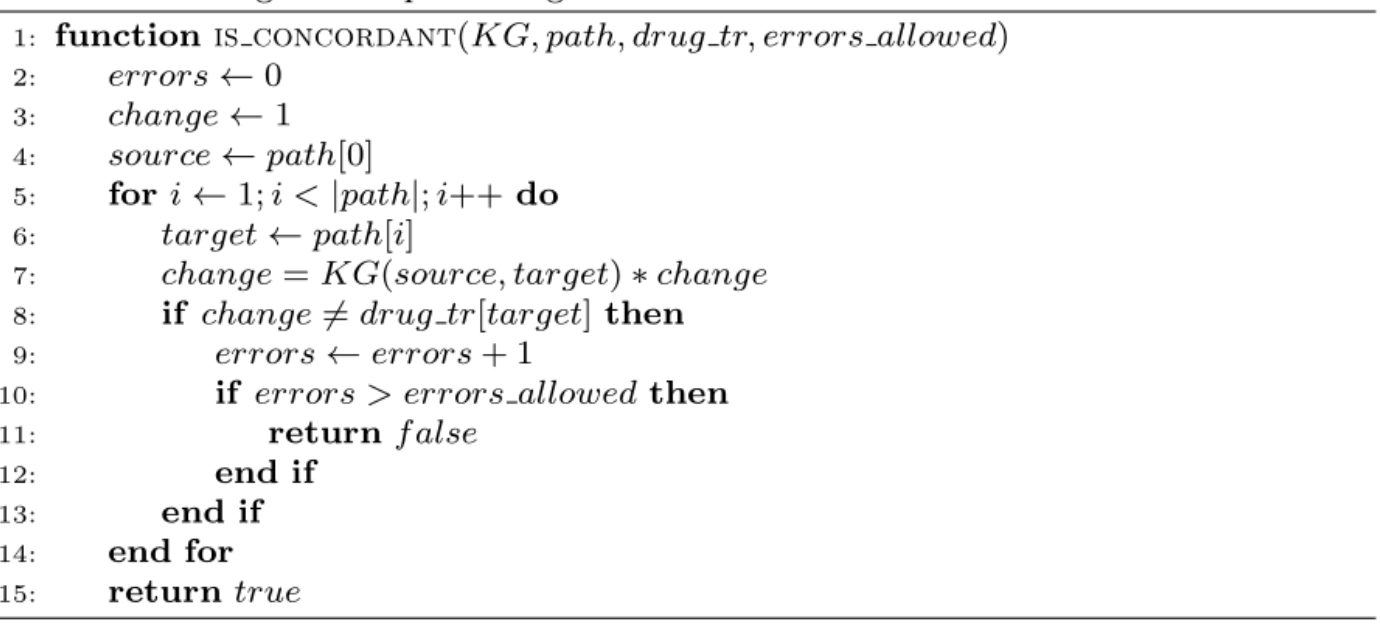




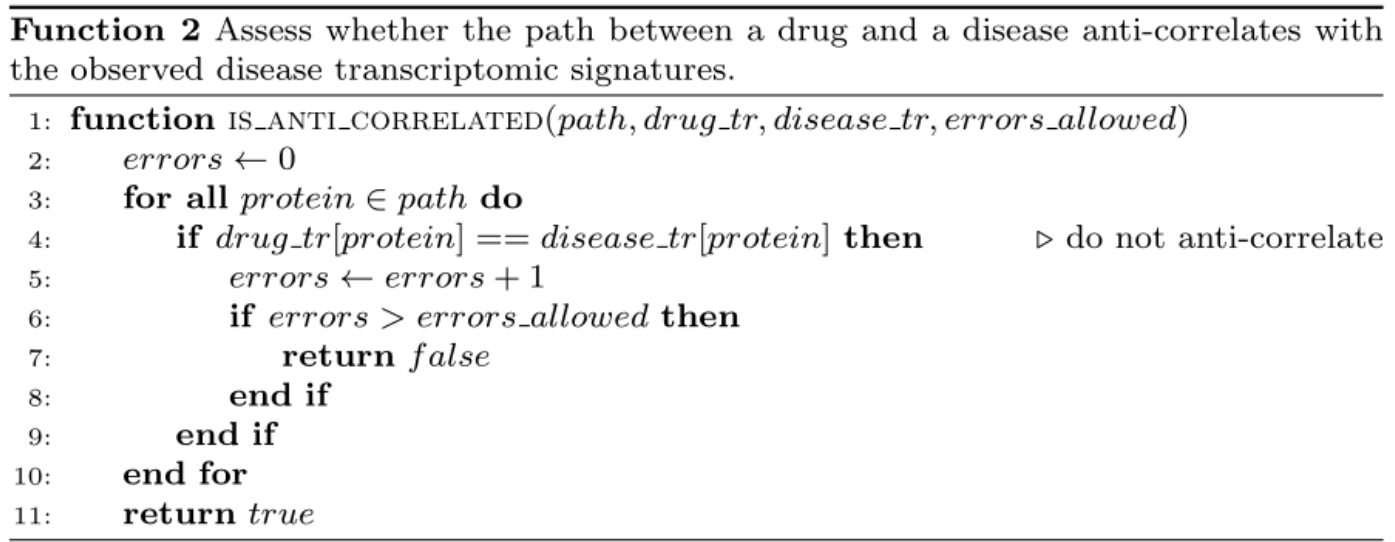

Figure 2. Pseudocode of the RPath algorithm. The core function of the algorithm, is_drug_prioritized, prioritizes drug candidates if there are paths between a drug-disease pair in the KG that are concordant or anti-correlated with observed transcriptomic signatures. The evaluation of the paths between a drug and disease which are concordant with the signatures observed in a drug-perturbed transcriptomic experiment is conducted via Function 1, is_concordant. Similarly, Function 2, is_anti_correlated, evaluates paths between a drug and disease which are anti-correlated with the signatures observed in a disease-specific transcriptomic experiment. Paths which both match the drug-perturbed signatures and contradict disease-specific signatures are then returned by RPath as promising drug candidates.

\subsection{Datasets and validation}

In this subsection, we present drug-perturbed and disease-specific transcriptomic datasets as well as the KGs used to demonstrate our methodology. We then introduce the strategy we follow to validate our methodology.

\subsubsection{Drug-perturbed and disease transcriptomic datasets}

We identified four databases that were suitable for our approach (Supplementary Table 1); drug-perturbed transcriptomic data were obtained from CREEDS (Wang et al., 2016) and L1000 (Subramanian et al., 2017) while disease transcriptomic data were collected from Open Targets (Ochoa et al., 2021) and GEO (Barrett et al., 2012). All experimental datasets from these resources (downloaded on 15.02.2021) were from gene expression changes measured in humans. Chemicals and diseases from datasets obtained from these databases were then mapped to PubChem compound identifiers and the Mondo Disease Ontology (MONDO), respectively, for consistency with the entities of the knowledge graphs presented in the next section. Similarly, gene identifiers in all datasets were harmonized to ENTREZ. Of the four databases, datasets from L1000 contained a binarized value for the direction of dysregulation for every gene (i.e., up-regulation and down-regulation), while for the remaining databases, fold changes were binarized for significantly dysregulated genes using $\mid \log _{2}$ fold change $\mid=1$ as a cutoff (Supplementary Text 1). Finally, we conducted a systematic search for databases that contained either a large number of drug-perturbed or disease-specific transcriptomic datasets. While this search initially resulted in 27 candidate databases (see Supplementary Table 1 for details about each dataset), the majority of them were not suitable for our study as they either contained too few transcriptomic datasets or the drugs/diseases in these datasets were not in the KGs used to demonstrate our methodology.

\subsubsection{Knowledge Graphs}


We demonstrate our methodology using two publicly available KGs that contain causal relations across drug, proteins, and diseases: OpenBioLink KG (Breit et al., 2020) and a custom KG (Rivas-Barragan et al., 2020). Both KGs are originally generated from a compedia of independent databases; thus, containing unique causal interactions depending on the source databases they include. As outlined in the algorithm, the KGs are reduced to encompass three types of causal edges: drug-protein (i.e., drug activates/inhibits protein), protein-protein (i.e., protein activates/inhibits protein), and protein-disease (i.e., protein activates/inhibits disease). Furthermore, the original node identifiers for chemicals and diseases in both KGs were respectively mapped to PubChem compound identifiers and MONDO to be consistent with the transcriptomic datasets. Next, we removed drugs and diseases that were not present in any of the four transcriptomic datasets presented in the previous subsection as the paths between these drug-disease pairs cannot be validated. Figure 3 shows the final statistics of both KGs after the previously outlined filtering steps. Supplementary Table 2 summarizes the overlap between the genes measured in each of the four transcriptomic datasets and their corresponding protein nodes in the KGs.
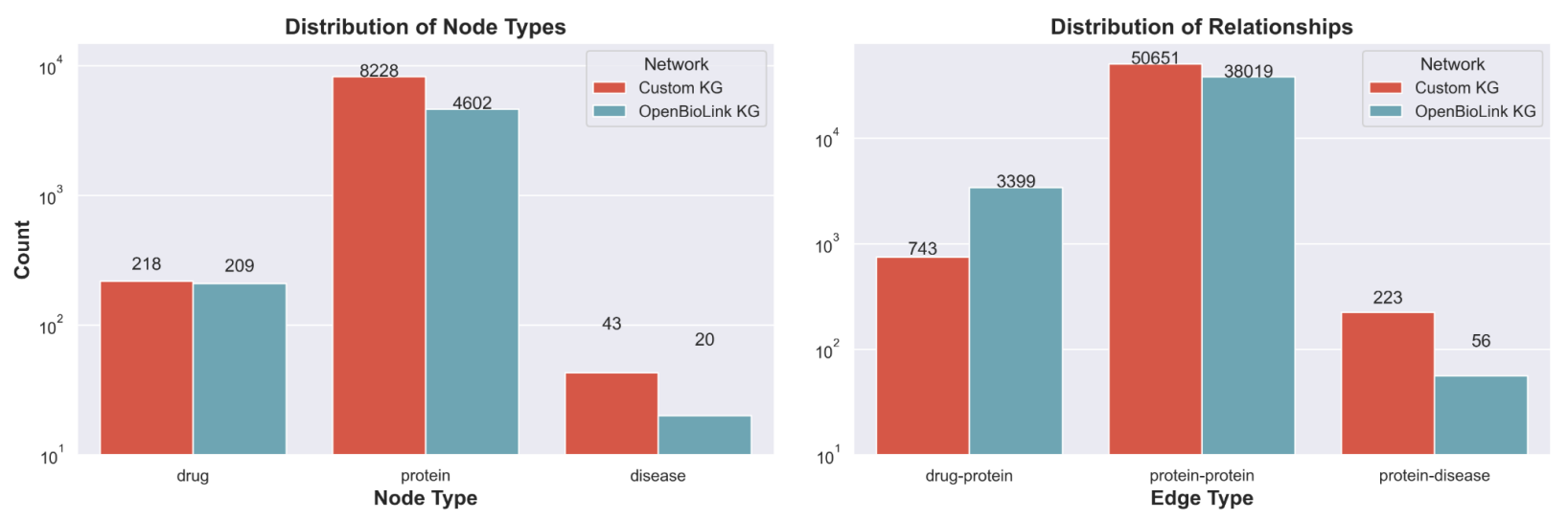

Figure 3. Distribution of node and edge types in the custom and OpenBioLink KGs. A visualization of the KGs can be found in Supplementary Figure 2 while properties of each of the two networks are detailed in Supplementary Table 4.

\subsubsection{Validation}

In line with other network-based approaches designed for drug discovery (Malas et al., 2019; Rivas-Barragan et al., 2020; Gysi et al., 2021), we used drug-disease pairs that have been clinically investigated as positive labels, extracting this information from ClinicalTrials.gov (accessed on 28.09.2020). Since drugs and diseases in ClinicalTrials.gov are formalized using MeSH identifiers, we harmonized these identifiers to the ontologies used in the KG (i.e., PubChem compound for chemicals and MONDO for diseases) using proprietary harmonization scripts. After the harmonization, any drug or disease in the KGs that was not present in any clinical trial or did not have any path to any disease in the KG was subsequently removed, as its corresponding node cannot be used in the presented validation.

To test the robustness of RPath in identifying these clinically investigated drug-disease pairs, we conducted eight independent analyses, one for each of the combinations of the two drug datasets, the two disease datasets, and the two KGs (e.g., CREEDS-GEO-OpenBioLink, LINCS-GEO-OpenBioLink, etc.). For each of these eight 
analyses, we ran RPath over a given KG to identify prioritized drug-disease pairs, in other words, pairs whose paths are both correlated with drug-perturbed transcriptomic signatures and anti-correlated with disease transcriptomic signatures. Here, we make two assumptions in the design of the algorithm. Firstly, paths with cycles or a length greater than 7 edges between a given drug and disease are not considered, assuming that the effects exerted by paths beyond this length are less biologically relevant (Rivas-Barragan et al., 2020). Secondly, we allow for at most one error between the transcriptomic data and a given path (see algorithm pseudocode in Supplementary Figure 1 for details). In this set of prioritized drug-disease pairs, we expect to retrieve a larger proportion of clinically investigated drug-disease pairs (i.e., positive labels) than expected by chance (i.e., proportion of positive labels in the dataset). Here, it is important to note that i) as in any drug discovery task, there is a class label imbalance (i.e., the vast majority of the drug-disease pairs are negative labels), and ii) since each dataset contains a different set of chemicals/diseases, each of the eight analyses has a different proportion of positive labels (between $1 \%$ and $15 \%$ ) (Supplementary Table 3). Furthermore, this type of validation falls into the so-called early retrieval problem. In other words, from the thousands of drug-disease pairs that are tested, we are exclusively prioritizing the top-ranked pairs that have been equally prioritized by the algorithm. This small subset represents the interesting drug-disease pairs that would be further investigated in the drug discovery process. In such cases, it is inadequate to apply metrics such as receiver operating characteristic (ROC) curves (i.e.. AUC-ROC and AUC-PR) as they operate on a full ranked list. Therefore, it does not necessarily evaluate the ability of a model to prioritize the most promising drug-disease pairs candidates (Berrar and Flach, 2012). Additionally, considering that not all drug-disease pairs have been clinically studied, a number of the negative labels might be falsely classified as positive. To address these issues, we evaluated RPath based on the ratio of true positives that appear in the prioritized drug-disease pairs (i.e., precision) and assessed whether the prioritized drug-disease pairs found through the algorithm contain a larger proportion of positive pairs than expected on average by chance.

As an additional validation, we benchmarked RPath against 11 equivalent approaches that can be used to prioritize drug-disease pairs based solely on network structure, as outlined by Abbas et al. (2021), Coşkun and Koyutürk (2021) and Zietz et al. (2020) (Supplementary Text 2).

\subsection{Implementation details}

The RPath algorithm and the benchmarked methods are implemented in Python leveraging NetworkX (v2.5) (https://networkx.github.io). Network visualizations were done using WebGL, D3.js, Three.js, Matplotlib and igraph. Source code, documentation, and data are available at https://github.com/enveda/RPath. The validation presented in the paper can be reproduced by running the Jupyter notebooks available at https://github.com/enveda/RPath/tree/master/src/notebooks. 


\section{Results}

This section is divided into three subsections that outline the different applications of RPath presented in this manuscript. In subsection 3.1, we demonstrate how RPath can be used to identify potential drug candidates for various diseases using a variety of KGs and datasets, outperforming numerous link prediction methods. Then, in subsection 3.2, we leverage the inherent interpretability of KGs to generate hypotheses for the predictions made by RPath. Finally, subsection 3.3 outlines how RPath can be reversed-engineered and alternatively used to predict targets for a given disease.

\subsection{Identification of drug candidates}

To demonstrate the ability of our algorithm to accurately identify drug candidate for a given disease, we evaluated its performance to recover clinically investigated drug-disease pairs using two distinct KGs and four transcriptomic datasets (i.e., two each containing numerous drug-perturbed and disease transcriptomic experiments). In this task, RPath consistently prioritized a significantly larger number of clinically investigated drug-disease pairs across all datasets and in both KGs compared with the precision expected by chance (i.e., probability of randomly picking a true positive among all possible drug-disease combinations) (Table 1). The highest precision values were found for the L1000-GEO datasets with $80 \%$ and $66.67 \%$ for the OpenBioLink and custom KGs, respectively. In the remaining datasets, the precision was approximately 50\%, except for the CREEDS-Open Target datasets in the custom KG that exclusively yielded a single drug-disease pair which was not in clinical trials. While the precision expected by chance approximately varied between $10 \%$ and $35 \%$, RPath consistently achieved higher precision values across nearly all datasets and KGs, ranging between $50 \%$ and $80 \%$ (e.g., more than five times higher for the L1000-GEO dataset in OpenBioLink running RPath (80\%) vs. chance (15.66\%)). Notably, the number of prioritized drug-disease pairs were constrained for two reasons: i) RPath requires transcriptomic information for a given drug and disease and, ii) the pair must also be present in the KG (see Supplementary Table 3 for details). Furthermore, apart from the low number of drug-disease pairs that fulfilled these criteria, RPath filters the majority of the drug-disease pairs with paths between them after overlaying the transcriptomic signatures in Steps 1 and 2 (see Figure 1) of the algorithm (Supplementary Table 5). For example, in the case of the CREEDS-GEO datasets and the OpenBioLink KG, the total number of diseases was 10 , resulting in only a couple of drug-disease pairs being prioritized. Nonetheless, we were still able to validate our methodology across multiple datasets and KGs, observing that RPath performed significantly better than chance at identifying clinically investigated drug-disease pairs.

\begin{tabular}{|c|c|c|c|c|}
\hline- & \multicolumn{2}{|c|}{ OpenBioLink KG } & \multicolumn{2}{|c|}{ Custom KG } \\
\hline Dataset combination & Precision $(\mathrm{TP} / \mathrm{TP}+\mathrm{FP})$ & $\begin{array}{c}\text { Expected precision by } \\
\text { chance }\end{array}$ & Precision $(\mathrm{TP} / \mathrm{TP}+\mathrm{FP})$ & $\begin{array}{c}\text { Expected precision by } \\
\text { chance }\end{array}$ \\
\hline
\end{tabular}




\begin{tabular}{|c|r|r|r|r|}
\hline L1000-GEO & $80 \%(4 / 5)$ & $15.66 \%$ & $66.67 \%(2 / 3)$ & $12.83 \%$ \\
\hline L1000-Open Target & $54.55 \%(6 / 11)$ & $11.32 \%$ & $50 \%(2 / 4)$ & $9.23 \%$ \\
\hline CREEDS-Open Target & $50 \%(1 / 2)$ & $26.17 \%$ & $0 \%(0 / 1)$ & $24.36 \%$ \\
\hline CREEDS-GEO & $50 \%(1 / 2)$ & $36.77 \%$ & $50 \%(1 / 2)$ & $33.52 \%$ \\
\hline
\end{tabular}

Table 1. Evaluation of RPath in multiple datasets across the two KGs using precision. Each row corresponds to the results of running RPath on a specific drug-disease dataset combination. The second and fourth columns show the performance that is expected to be achieved by chance.

Finally, we benchmarked RPath against 11 alternative methods that have been used to predict drug-disease links in a KG with the same characteristics as the ones used in this work (Abbas et al., 2021; Zietz et al., 2020). The precision of these methods varied between 5\% and 43\% (Supplementary Table 6). Furthermore, since the majority of these methods prioritize a drug and a disease based on their network proximity (e.g., shortest paths and number of shared nodes), these methods recurrently prioritized the same set of drug-disease pairs. Thus, these methods could not be used to prioritize drugs outside the vicinity of disease-associated proteins since only a minority of drug-disease pairs are connected by a single protein, but the majority of them contain longer paths that are not considered by these methods (Supplementary Table 5).

\subsection{Interpretation of the mechanisms of action of the proposed drug candidates}

Here, we explore the paths between two of the validated drug-disease pairs that were prioritized by RPath to demonstrate how our approach can be used to deconvolute the mechanism of action of a given drug (Figure 4). We deliberately selected bicalutamide and ponatinib, two anti-cancer drugs already approved for prostate cancer and acute myeloid leukemia, respectively. The reason is that the mechanisms of action of these drugs have been widely studied and allow us to compare the mechanistic paths identified by RPath against known interactions and pathways reported in scientific literature.

First, we investigated ponatinib, a multi-targeted tyrosine-kinase inhibitor, which is used to treat acute myeloid leukaemia (AML) (Figure 4a). Among the targets of this drug present in the concordant paths for this pair, we were able to identify fms-like tyrosine kinase 3 (FLT3), which is mutated in approximately $20 \%$ of AML patients (Smith et al., 2012) and several members of the FGFR family proteins. Furthermore, we observed other proteins including KDR, LYN, and SRC, all of which are kinase-associated targets in AML. As a downstream target of these proteins, we found JAK2, a well-studied player in myeloproliferative diseases, with known mutations and hypermethylation events. We further identified the transcription factor, CEBPA, that is critical for normal development of granulocytes and is also implicated in AML (Pabst and Mueller, 2009) and the SPI1 gene, from which circSPI1, a circular RNA derived from the gene, has recently been shown to be highly expressed in AML patients (Wang et al., 2021). Other proteins that are inhibited as a result of the signaling cascade triggered by ponatinib include KIT, which is implicated in cell death in AML (Heo et al., 2017). RAS family members NRAS and KRAS, both of which are 
associated with the prognosis of solid tumors and hematological malignancies, including AML (Mascaux et al., 2005) were also implicated.

The second studied drug-disease pair is bicalutamide (brand name Casodex ${ }^{\mathrm{TM}}$ ), used for the treatment of prostate cancer. Bicalutamide is an anti-androgen medication that binds to the androgen receptor (AR), as illustrated in Figure 4b. The paths between bicalutamide and prostate cancer point to several downstream targets of this drug, including the epigenetic regulator KMT2D, which is known to sustain prostate carcinogenesis by epigenetic mechanisms (Ly et al., 2009), and NECAB3, known to enhance the activity of HIF1A, thus promoting glycolysis under normoxic conditions and enhancing tumorigenicity in cancer cells (Nakaoka et al., 2016). Furthermore, we were able to identify CTNNB1, which plays a role in the development of numerous prostate cancers (Gerstein et al., 2002). Interestingly, we also observed novel players that have not yet been reported in the literature, such as GNAI1, SYMPK, UBR5, and MEF2C that may provide new insights on the mechanism of action of this drug.

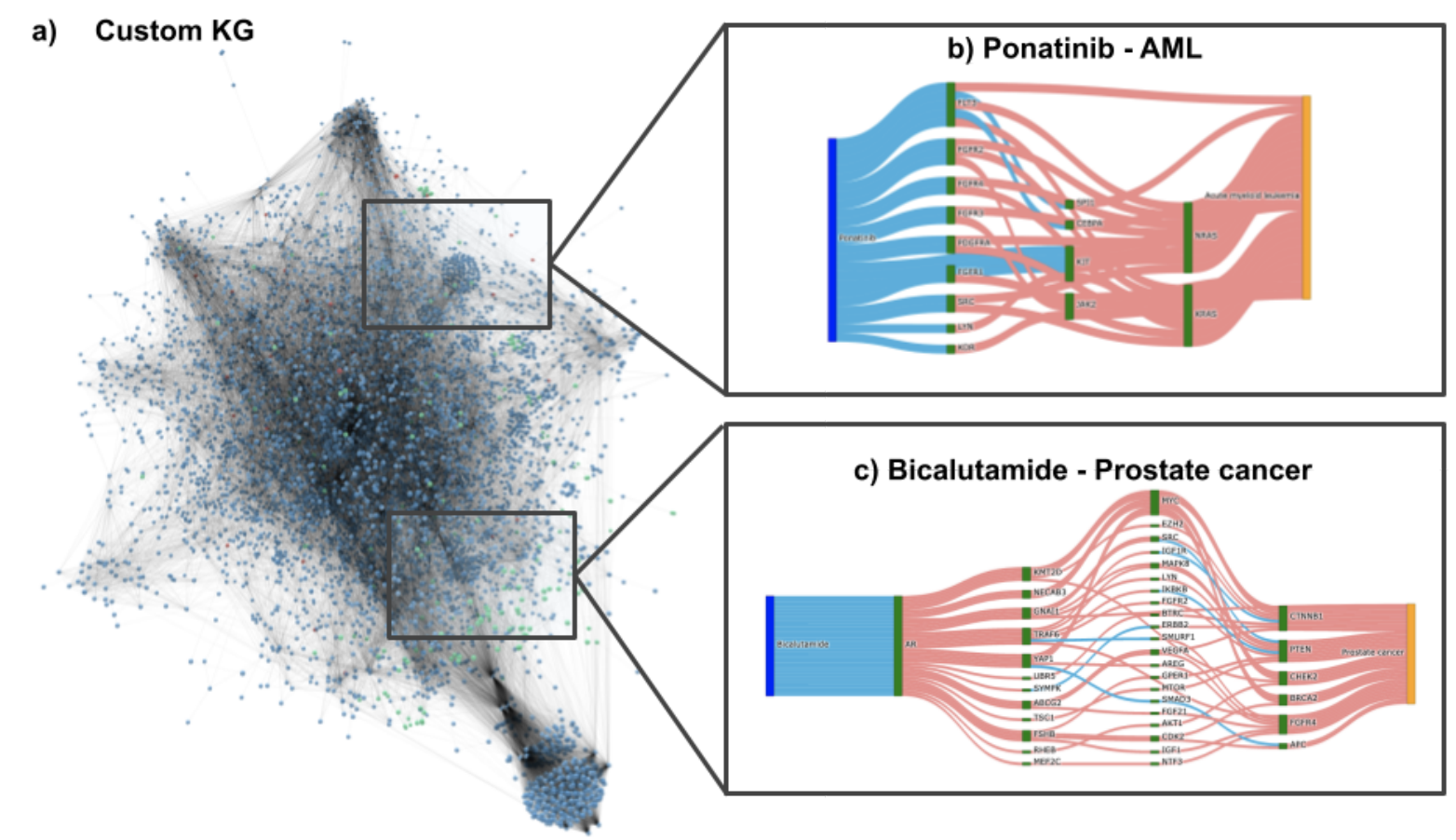

Figure 4. Devoncoluting the mechanism of action of a drug through RPath. By investigating all the paths of a given length between a drug and a disease in a KG, we can analyze the different mechanisms that are proposed by RPath. a) Visualization of the custom KG. Proteins are colored in blue, diseases in red and chemicals in green. Sankey diagram illustrating a sample of the paths between ponatinib and AML (b) and bicalutamide and prostate cancer (c) for the custom KG. Activatory relations in the Sankey diagrams are colored in red and inhibitory relations in blue.

\subsection{Target prioritization}

Prior to the identification of a therapeutic drug candidate for any given disease, a crucial first step is to often identify biologically relevant protein targets. Ideally, the perturbation of a particular protein target in a disease state should 
result in the reversal of the observed phenotype. In a similar manner to the above-mentioned applications, by reasoning over the KG guided by disease signatures, RPath can be used for target prioritization. Since, as per our knowledge, there are no large datasets that contain information about known targets for a wide variety of indications, we could not conduct a validation strategy similar to the analyses presented in subsection 3.1. Instead, we focused on evaluating the top prioritized protein targets across all diseases using literature evidence (Table 2).

Among the top protein target-disease pairs proposed by RPath, two have already been associated with AML, including PRKCA, for which several drugs already exist (Konopatskaya and Poole, 2010; Takami, M et al., 2018), and CXCL8/IL-8 (Campbell et al., 2013; Schinke et al., 2015; Kuett et al., 2015). Furthermore, CDC42, which has been proposed as a candidate target for medulloblastoma, plays a role in several cancers. Specifically, CDC42 has been shown to act as a regulator of medulloblastoma-associated genes (Nalla et al., 2010) and compounds for its inhibition have also been proposed (Hong et al., 2013).

\begin{tabular}{|l|l|r|r|l|l|}
\hline Protein target & Disease & Concordant paths & $\begin{array}{l}\text { Nodes in the concordant } \\
\text { paths }\end{array}$ & KG & $\begin{array}{l}\text { Transcriptomic } \\
\text { dataset }\end{array}$ \\
\hline NOG & AML & 18,456 & 1,008 & Custom KG & GEO \\
\hline PRKCA & AML & 12,861 & 669 & Custom KG & GEO \\
\hline CXCL8 / IL-8 & AML & 7,234 & 465 & Custom KG & GEO \\
\hline NOG & Plasma cell myeloma & 5743 & 616 & Custom KG & GEO \\
\hline CDC42 & Medulloblastoma & 5,651 & 91 & OpenBioLink & GEO \\
\hline
\end{tabular}

Table 2. Top 5 prioritized protein target-disease pairs. These results were obtained by running RPath over both KGs with the GEO and Open Targets datasets using the same path length as the drug discovery task (see Methods). Pairs were prioritized based on the number of concordant paths. The vast majority of pairs were prioritized using the disease transcriptomic signatures from the GEO dataset given its larger coverage of measured genes compared to Open Targets (Supplementary Table 2).

\section{Discussion}

In this work, we present a novel methodology that leverages prior knowledge from causal relations across multiple biological modalities in KGs and assesses their concordance with transcriptomic signatures for drug discovery. In the past, several algorithms have been primarily introduced for the interpretation of transcriptomic signatures by reasoning over shortest paths (Chindelevitch et al., 2012; Krämer et al., 2014) or bipartite graphs (Catlett et al., 2013; Martin et al., 2012; Martin et al., 2014). Though these algorithms could also be indirectly applied for drug discovery, they present some shortcomings: i) they operate on homogeneous causal graphs with a single entity type (e.g., protein nodes), ii) they are solely conducted on single contrast experiments (e.g., drug-treated vs. control), and iii) they do not fully exploit all possible paths in these causal graphs. RPath addresses these shortcomings by reasoning over all possible causal paths in a multimodal $\mathrm{KG}$ and leveraging both drug and disease transcriptomics signatures. First, our algorithm reasons over the ensemble of paths between a given drug and a disease in a KG. Second, it evaluates the concordance of these paths against the transcriptomic changes experimentally observed for 
that drug. Next, it assesses whether the effect of these paths is opposite to the transcriptomic signatures observed within the disease context. In a final step, the algorithm identifies potential drug candidates as those whose paths correlate with drug-perturbed transcriptomic signatures and are anti-correlated to the disease transcriptomic signatures. We have validated our methodology in eight independent analyses, finding that RPath consistently identifies a large proportion of clinically investigated drug-disease pairs over multiple datasets and KGs. Additionally, we conducted several robustness experiments and benchmarked the algorithm against 11 network-based methodologies. Finally, we also showed how our approach can be used to deconvolute the mechanism of action of a drug as well as to prioritize protein targets for a given disease.

We acknowledge a few shortcomings in our work that are worth discussion. Firstly, we were limited by the availability of high-quality annotated transcriptomic datasets for drugs and diseases, as only four of the approximately 30 datasets that we identified met our requirements. Furthermore, the coverage of measured genes varied largely across experiments. For instance, while the average number of genes measured in the Open Targets dataset (Ochoa et al., 2021) was approximately 900, that number dropped to 500 in the CREEDS dataset (Wang et al., 2016) (Supplementary Table 2). In contrast, the total number of proteins in the KGs were in the range of several thousands. As RPath requires that signatures from both these drug and disease datasets be mapped to the KG, most of the proteins in the KGs could not be quantified. Thus, we allowed for up to one error when calculating the anti-correlation in the path between a drug and a disease. We expect that this limitation will be overcome by high-quality, consistent datasets such as those generated in large pharmaceutical enterprises and emerging data-driven biotech companies looking to leverage large-scale computational technologies. Furthermore, two other reasons justified an error within the path. Firstly, introducing an error limits the impact of an arbitrary fold change cut-off, which ultimately determines the up-/down-regulation of each protein. Secondly, some paths might contain causal relations that do not reflect a change at the transcription level of the affected protein (e.g., phosphorylation of a protein kinase) (Hill et al., 2017; Babur et al., 2021). Another characteristic of our approach is that the identification of a potential drug for a given disease requires knowledge of the protein target and the effect of the drug on it. However, this information is not always available or must be inferred using computational approaches.

While we have demonstrated our novel algorithm across multiple datasets and KGs, we envision multiple other applications. Firstly, by incorporating time series data into the analysis, we can determine how the paths between the drug and the disease are altered over time following the concept outlined by Coker et al. (2017). Additionally, although we have demonstrated our methodology using transcriptomic data, other modalities can be used if the KG contains causal relations for these entities (e.g., metabolomics). Furthermore, although a multimodal KG may lack the context within which each relation occurs, RPath inherently takes this into account by removing the paths which do not match the observed transcriptomic signatures. However, the algorithm could also be applied on a disease-specific KG in order to model the pathophysiological mechanisms characteristic of a given phenotype (Boué et al., 2015; Domingo-Fernández et al., 2017). 


\section{References}

1. Abbas, K., et al. (2021). Application of network link prediction in drug discovery. BMC Bioinformatics, 22(1), 1-21. https://doi.org/10.1186/s12859-021-04082-y

2. Babur, Ö., et al. (2021). Causal interactions from proteomic profiles: molecular data meets pathway knowledge. Patterns 2, 100257. https://doi.org/10.1016/i.patter.2021.100257

3. Barrett, T., Wilhite, S. E., Ledoux, P., Evangelista, C., Kim, I. F., et al. (2012). NCBI GEO: archive for functional genomics data sets-update. Nucleic acids research, 41(D1), D991-D995. https://doi.org/10.1093/nar/gks1193

4. Belyaeva, A., et al., C. (2021). Causal network models of SARS-CoV-2 expression and aging to identify candidates for drug repurposing. Nature communications, 12(1), 1-13. https://doi.org/10.1038/s41467-021-21056-Z

5. Berrar, D, and Flach, P. (2012). Caveats and pitfalls of ROC analysis in clinical microarray research (and how to avoid them). Briefings in bioinformatics, 13(1), 83-97. https://doi.org/10.1093/bib/bbr008

6. Bharadhwaj, V. S., et al. (2021). CLEP: A Hybrid Data-and Knowledge-Driven Framework for Generating Patient Representations. Bioinformatics, btab340. https://doi.org/10.1093/bioinformatics/btab340

7. Bonner, S., et al. (2021). A Review of Biomedical Datasets Relating to Drug Discovery: A Knowledge Graph Perspective. arXiv preprint arXiv:2102.10062.

8. Boué, S., et al. (2015). Causal biological network database: a comprehensive platform of causal biological network models focused on the pulmonary and vascular systems. Database, bav030. https://doi.org/10.1093/database/bav030

9. Breit, A, Ott, S, Agibetov, A, and Samwald, M. (2020). OpenBioLink: A resource and benchmarking framework for large-scale biomedical link prediction. Bioinformatics, 36(13), 4097-4098, https://doi.org/10.1093/bioinformatics/btaa274

10. Campbell, L. M., Maxwell, P. J., and Waugh, D. J. (2013). Rationale and means to target pro-inflammatory interleukin-8 (CXCL8) signaling in cancer. Pharmaceuticals, 6(8), 929-959. https://doi.org/10.3390/ph6080929

11. Catlett, N. L., et al. (2013). Reverse causal reasoning: applying qualitative causal knowledge to the interpretation of high-throughput data. BMC Bioinformatics, $14(1), 340$. https://doi.org/10.1186/1471-2105-14-340

12. Chindelevitch, L., et al. (2012). Causal reasoning on biological networks: interpreting transcriptional changes. Bioinformatics, 28(8), 1114-1121. https://doi.org/10.1093/bioinformatics/bts090

13. Coker, E. A., Mitsopoulos, C., Workman, P., and Al-Lazikani, B. (2017). SiGNet: A signaling network data simulator to enable signaling network inference. Plos one, 12(5), e0177701. https://doi.org/10.1371/journal.pone.0177701

14. Coşkun, M., and Koyutürk, M. (2021). Node Similarity Based Graph Convolution for Link Prediction in Biological Networks. Bioinformatics, btab464, https://doi.org/10.1093/bioinformatics/btab464

15. Domingo-Fernández, D., et al. (2017). Multimodal mechanistic signatures for neurodegenerative diseases (NeuroMMSig): a web server for mechanism enrichment. Bioinformatics, 33(22), 3679-3681. https://doi.org/10.1093/bioinformatics/btx399 
16. Emon, M. A., Domingo-Fernández, D., Hoyt, C. T., and Hofmann-Apitius, M. (2020). PS4DR: A multimodal workflow for identification and prioritization of drugs based on pathway signatures. $B M C$ Bioinformatics, 21, 1-21. https://doi.org/10.1186/s12859-020-03568-5

17. Fotis, C., Antoranz, A., Hatziavramidis, D., Sakellaropoulos, T., and Alexopoulos, L. G. (2018). Network-based technologies for early drug discovery. Drug discovery today, 23(3), 626-635. https://doi.org/10.1016/j.drudis.2017.12.001

18. Gerstein, A.V., et al. (2002). APC/CTNNB1 ( $\beta$-catenin) pathway alterations in human prostate cancers. Genes, Chromosomes and Cancer, 34(1), 9-16. https://doi.org/10.1002/gcc.10037

19. Gysi, D. et al. (2021). Network medicine framework for identifying drug repurposing opportunities for COVID-19. Proceedings of the National Academy of Sciences, 118 (19) e2025581118. https://doi.org/10.1073/pnas.2025581118

20. Heo, S.K., et al. (2017). Targeting c-KIT (CD117) by dasatinib and radotinib promotes acute myeloid leukemia cell death. Scientific reports, 7(1), 1-12. https://doi.org/10.1038/s41598-017-15492-5

21. Hill, S. M., et al. (2017). Context specificity in causal signaling networks revealed by phosphoprotein profiling. Cell systems, 4(1), 73-83. https://doi.org/10.1016/i.cels.2016.11.013

22. Hong, L., et al. (2013). Characterization of a Cdc42 protein inhibitor and its use as a molecular probe. Journal of Biological Chemistry, 288(12), 8531-8543. https://doi.org/10.1074/ibc.M112.435941

23. Iorio, F., et al. (2010). Discovery of drug mode of action and drug repositioning from transcriptional responses. Proceedings of the National Academy of Sciences, 107(33), 14621-14626. https://doi.org/10.1073/pnas.1000138107

24. Konopatskaya, O., and Poole, A. W. (2010). Protein kinase C $\alpha$ : disease regulator and therapeutic target. Trends in pharmacological sciences, 31(1), 8-14. https://doi.org/10.1016/j.tips.2009.10.006

25. Krämer, A., Green, J., Pollard Jr, J., and Tugendreich, S. (2014). Causal analysis approaches in ingenuity pathway analysis. Bioinformatics, 30(4), 523-530. https://doi.org/10.1093/bioinformatics/btt703

26. Kuett, A., et al. (2015). IL-8 as mediator in the microenvironment-leukaemia network in acute myeloid leukaemia. Scientific reports, 5(1), 1-11. https://doi.org/10.1038/srep18411

27. Liu, A., Trairatphisan, P., Gjerga, E., Didangelos, A., Barratt, J., and Saez-Rodriguez, J. (2019). From expression footprints to causal pathways: contextualizing large signaling networks with CARNIVAL. npj Systems Biology and Applications, 5(1), 1-10. https://doi.org/10.1038/s41540-019-0118-Z

28. Ly, S., et al. (2018). Histone methyltransferase KMT2D sustains prostate carcinogenesis and metastasis via epigenetically activating LIFR and KLF4. Oncogene, 37(10), 1354-1368. https://doi.org/10.1038/s41388-017-0026-X

29. MacLean, F. (2021). Knowledge graphs and their applications in drug discovery. Expert Opinion on Drug Discovery, 1-13. https://doi.org/10.1080/17460441.2021.1910673

30. Malas, TB., et al. (2019). Drug prioritization using the semantic properties of a knowledge graph. Scientific reports 9.1: 1-10. https://doi.org/10.1038/s41598-019-42806-6

31. Martin, F., et al. (2012). Assessment of network perturbation amplitudes by applying high-throughput data to causal biological networks. BMC Systems biology, 6(1), 54. https://doi.org/10.1186/1752-0509-6-54 
32. Martin, F., et al. (2014). Quantification of biological network perturbations for mechanistic insight and diagnostics using two-layer causal models. BMC Bioinformatics, 15(1), 238. https://doi.org/10.1186/1471-2105-15-238

33. Mascaux, C., et al. (2005). The role of RAS oncogene in survival of patients with lung cancer: a systematic review of the literature with meta-analysis. British journal of cancer, 92(1), 131-139. https://doi.org/10.1038/sj.bjc.6602258

34. Nakaoka, H.J., et al. (2016). NECAB3 promotes activation of hypoxia-inducible factor-1 during normoxia and enhances tumourigenicity of cancer cells. Scientific reports, 6(1), 1-13. https://doi.org/10.1038/srep22784

35. Nalla, A. K., et al. (2010). Suppression of uPAR retards radiation-induced invasion and migration mediated by integrin $\beta 1 /$ FAK signaling in medulloblastoma. PloS one, 5(9), e13006. https://doi.org/10.1371/journal.pone.0013006

36. Nelson, W., et al. (2019). To embed or not: network embedding as a paradigm in computational biology. Frontiers in genetics, 10:381. https://doi.org/10.3389/fgene.2019.00381

37. Ochoa, D., et al. (2021). Open Targets Platform: supporting systematic drug-target identification and prioritisation. Nucleic Acids Research, 49(D1), D1302-D1310. https://doi.org/10.1093/nar/gkaa1027

38. Pabst, T. and Mueller, B.U. (2009). Complexity of CEBPA dysregulation in human acute myeloid leukemia. Clinical Cancer Research, 15(17), 5303-5307. https://doi.org/10.1158/1078-0432.CCR-08-2941

39. Peyvandipour, A., Saberian, N., Shafi, A., Donato, M., and Draghici, S. (2018). A novel computational approach for drug repurposing using systems biology. Bioinformatics, 34(16), 2817-2825. https://doi.org/10.1093/bioinformatics/bty133

40. Rivas-Barragan, D, Mubeen, S, Bernat, FG, Hofmann-Apitius, M, and Domingo-Fernández, D. (2020). Drug2ways: Reasoning over causal paths in biological networks for drug discovery. PLoS computational biology, 16(12), e1008464. https://doi.org/10.1371/journal.pcbi.1008464

41. Samart, K., Tuyishime, P., Krishnan, A., and Ravi, J. (2021). Reconciling multiple connectivity scores for drug repurposing. Briefings in Bioinformatics, bbab161. https://doi.org/10.1093/bib/bbab161

42. Sang, S., et al. (2018). GrEDeL: A Knowledge Graph Embedding Based Method for Drug Discovery From Biomedical Literatures. IEEE Access, 7, 8404-8415. https://doi.org/10.1109/ACCESS.2018.2886311

43. Schaefer, M. H., Serrano, L., and Andrade-Navarro, M. A. (2015). Correcting for the study bias associated with protein-protein interaction measurements reveals differences between protein degree distributions from different cancer types. Frontiers in genetics, 6, 260. https://doi.org/10.3389/fgene.2015.00260

44. Schinke, C., et al. (2015). IL8-CXCR2 pathway inhibition as a therapeutic strategy against MDS and AML stem cells. Blood, 125(20), 3144-3152. https://doi.org/10.1182/blood-2015-01-621631

45. Sirota, M., et al. (2011). Discovery and preclinical validation of drug indications using compendia of public gene expression data. Science translational medicine, 3(96), 96ra77-96ra77. https://doi.org/10.1126/scitranslmed.3001318

46. Smith, C.C., et al. (2012). Validation of ITD mutations in FLT3 as a therapeutic target in human acute myeloid leukaemia. Nature, 485(7397), 260-263. https://doi.org/10.1038/nature11016

47. Subramanian, A., et al. (2017). A next generation connectivity map: L1000 platform and the first 1,000,000 profiles. Cell, 171(6), 1437-1452. https://doi.org/10.1016/j.cell.2017.10.049 
48. Takami, M., et al. (2018). Protein kinase C alpha-mediated phosphorylation of PIM-1L promotes the survival and proliferation of acute myeloid leukemia cells. Biochemical and biophysical research communications, 503(3), 1364-1371. https://doi.org/10.1016/i.bbrc.2018.07.049

49. Wang, Z., Monteiro, C. D., Jagodnik, K. M., Fernandez, N. F., Gundersen, G. W., Rouillard, A. D., et al. (2016). Extraction and analysis of signatures from the Gene Expression Omnibus by the crowd. Nature communications, 7(1), 1-11. https://doi.org/10.1038/ncomms12846

50. Wang, X., Jin, P., Zhang, Y. and Wang, K. (2021). CircSPI1 acts as an oncogene in acute myeloid leukemia through antagonizing SPI1 and interacting with microRNAs. Cell death \& disease, 12(4), 1-13. https://doi.org/10.1038/s41419-021-03566-2

51. Winkler, S. et al. (2021). de novo identification of maximally deregulated subnetworks based on multi-omics data with DeRegNet. bioRxiv 2021.05.11.443638 https://doi.org/10.1101/2021.05.11.443638

52. Zietz, M., et al. (2020). The probability of edge existence due to node degree: a baseline for network-based predictions. https://github.com/greenelab/xswap-manuscript

53. Zitnik, M., Agrawal, M., and Leskovec, J. (2018). Modeling polypharmacy side effects with graph convolutional networks. Bioinformatics, 34(13), i457-i466. https://doi.org/10.1093/bioinformatics/bty294

\section{Conflict of Interest}

We declare competing interests. DDF, YG, AP, CWD, BBM, DH, JR, and VC are employees of Enveda Biosciences Inc. during the course of this work and have real or potential ownership interest in Enveda Biosciences Inc. 


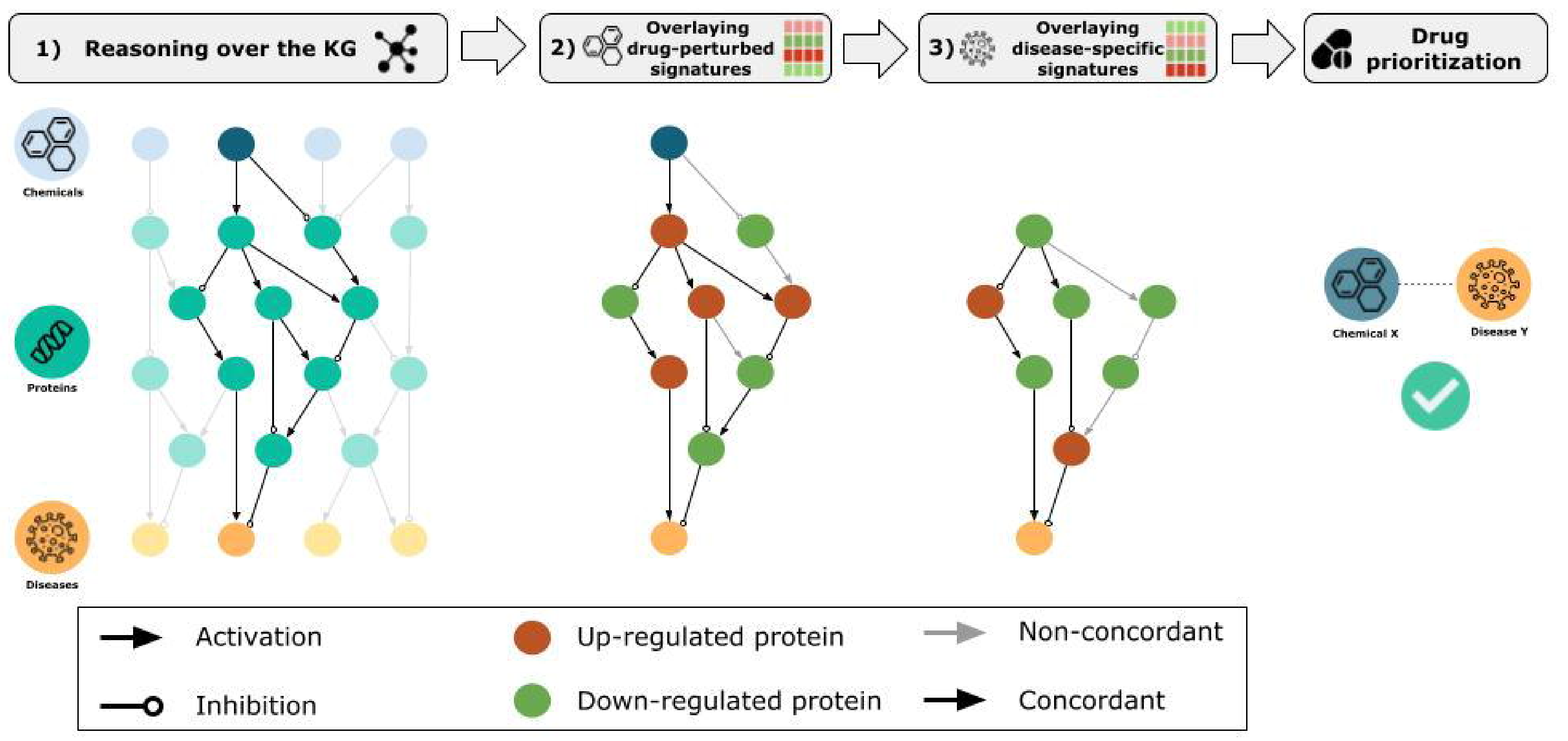


Distribution of Node Types

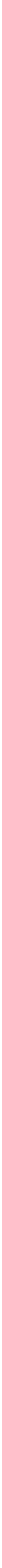




\section{a) Custom KG}
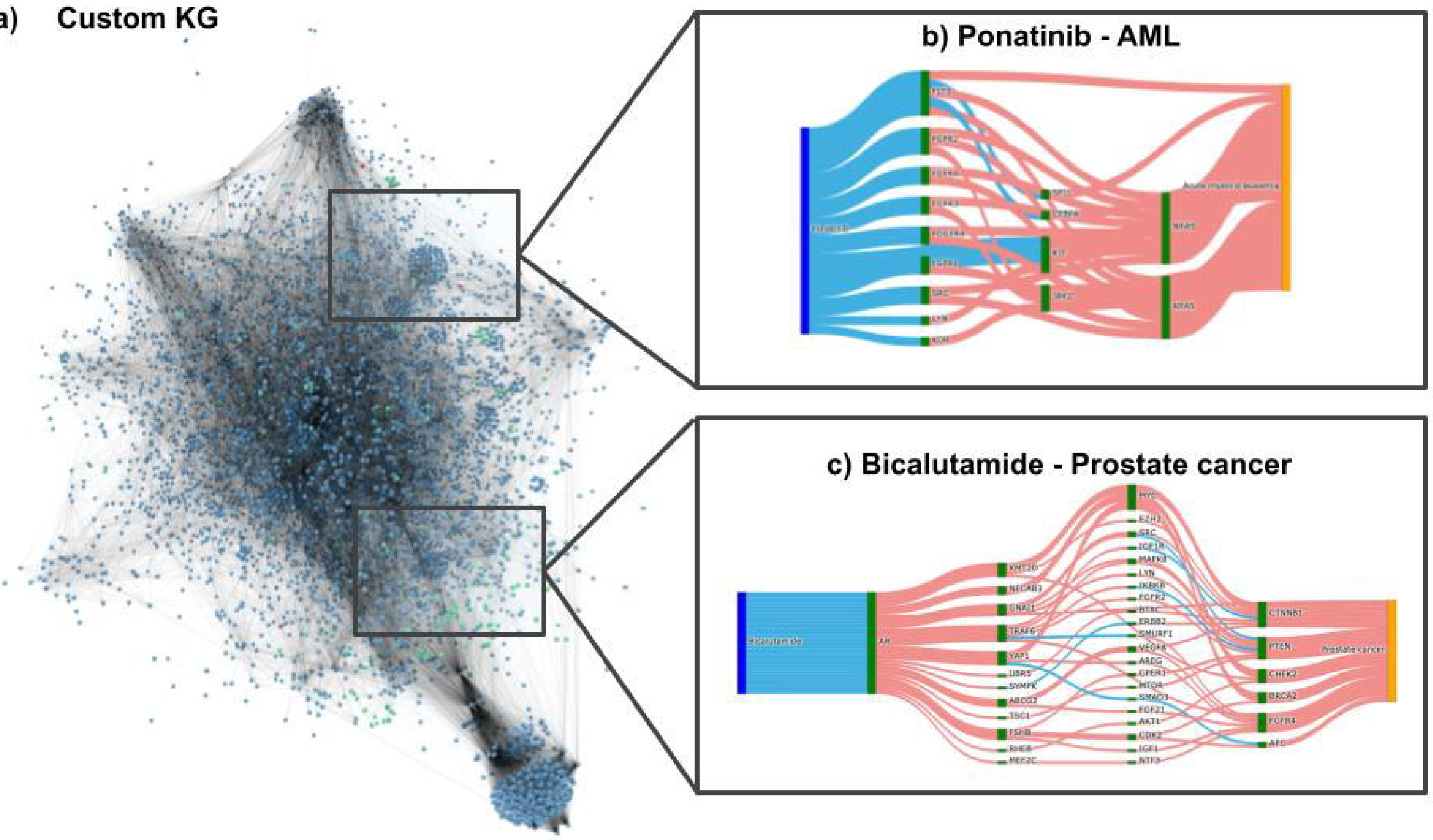

c) Bicalutamide - Prostate cancer

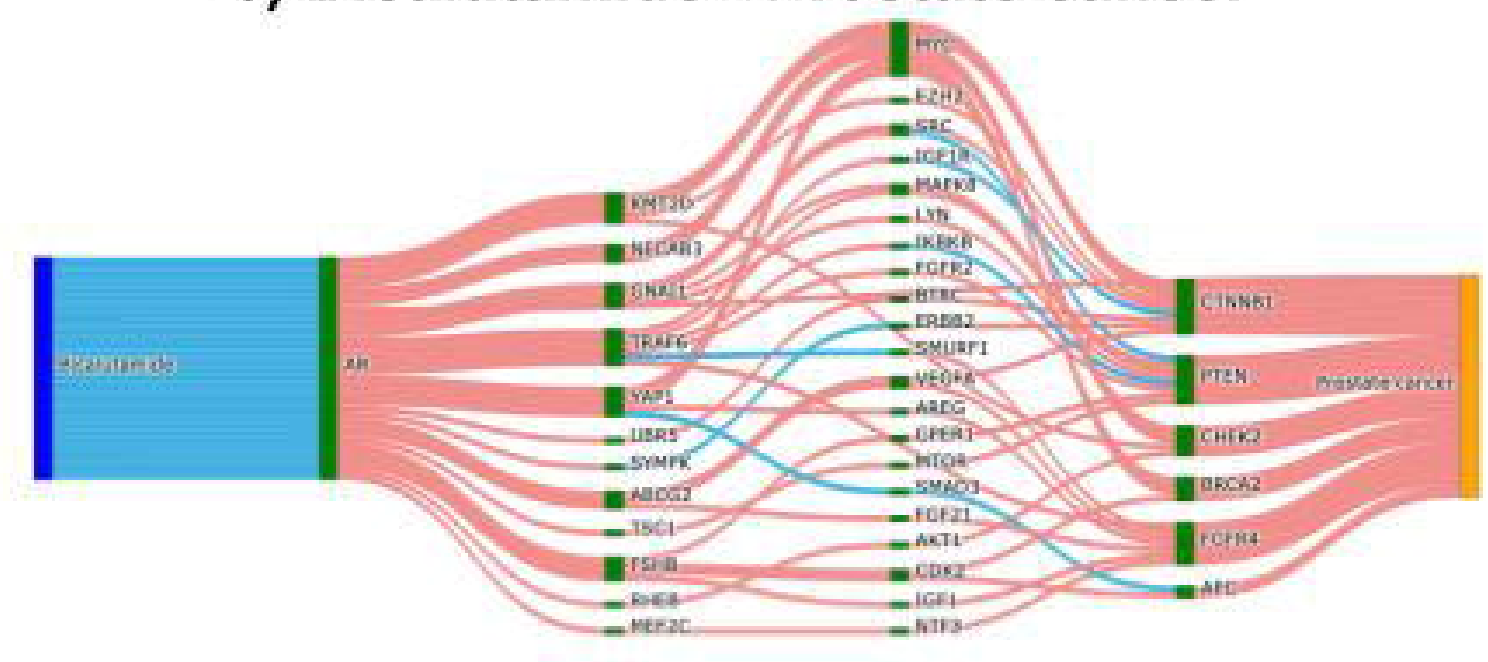

